\title{
The Human CD53 Gene, Coding for a Four Transmembrane Domain Protein, Maps to Chromosomal Region Ip13
}

M. Eugenia Gonzalez, Fernando Pardo-Manuel de Villena, Elena Fernandez-Ruiz, Santiago Rodriguez de Cordoba, and Pedro A. Lazo

Unidad de Genética Molecular (CSIC), Centro Nacional de Biología Celular y Retrovirus, Instituto de Salud Carlos III, 28220 Majadahonda, Madrid, Spain,

Centro de Investigaciones Biológicas, CSIC, Velazquez 144, and Servicio de Inmunolog[a, Hospital de la Princesa, Universidad Autónoma de Madrid, 28006 Madrid, Spain

The rat OX44/CD53 protein is the prototypic member of a "novel" family of proteins. These proteins are characterized by four highly hydrophobic transmembrane domains, two small extracellular domains, one of which is extensively $\mathrm{N}$-glycosylated, and both the amino and the carboxy terminus intracytoplasmic. The function of these proteins remains elusive and several possible functions have been suggested depending on the experimental system used, but all of them are somehow implicated in the control of cell proliferation by binding to an unknown ligand. In rat, CD53 antigen is detected on several mature cell types of the hematopoietic system, including macrophages, monocytes, granulocytes, leukocytes, and $\mathrm{B}$ and $\mathrm{T}$ cells, as well as osteoblasts and osteoclasts. In rat macrophages we have found that the CD53 protein also stimulates the production of nitric oxide (submitted for publication), which is implicated in septic and hemorraghic shocks. Preliminary data based on somatic cell hybrids indicated that the human CD53 gene is located on chromosome. To confirm these data and to determine precisely the location of human CD53 gene in chromosome 1, we have used fluorescence in situ hybridization (FISH). Using a rat OX-44 cDNA probe, a human genomic clone, hROX44, containing a 17-kb-long insert was isolated and demonstrated to include the complete human $O X-44 / C D 53$ gene. FISH was performed as previously reported. Briefly, peripheral blood lymphocytes were obtained from a human male by Lymphoprep gradient centrifugation and stimulated with phytohemagglutinin for $72 \mathrm{~h}$ Colchicine $(0.2$ $\mathrm{ttg} / \mathrm{ml}$ ) was added to the culture and kept for $1 \mathrm{~h}$. The X-hROX44 genomic clone was labeled by nick-translation with digoxigenin-11-dUTP. After $1 \mathrm{~h}$ of suppression hybridizition at $37^{\circ} \mathrm{C}$, hybridization was performed in $50 \%$ formamide containing $10 \%$ dextran sulfate, $2 \times \mathrm{SSC}$, and 50 mMphosphate, $\mathrm{pH} 7.0,2 \mathrm{ngl}$ of labeled probe, and a 500-fold excess of both Cot1 DNA (fast renaturation fraction of human genomic DNA) and sonicated human placental DNA at $37^{\circ} \mathrm{C}$ overnight. Washings and detection with TRITC-conjugated antibodies were performed as described. Chromosomes were counterstained with $75 \mathrm{ng} / \mathrm{ml}$ of 4',6-diamino-2phenylindole in antifade medium. After the fluorescence microscopy, GTG-banding was performed as described. A total of 35 metaphase spreads were analyzed for the presence of fluorescent spots; $89 \%$ of them showed hybridization signals on the pericentromeric region of the short arm of chromosome 1, in one $(39 \%)$ or both $(61 \%)$ chromatids (Fig. 1). Hybridization was highly specific, and no other chromosomes showed spots systematically. This nonrandom distribution was analyzed after GTGbanding of metaphases spreads to determine the precise location of the CD53 gene (Figs. 1A and 1B). The results of this analysis are summarized in Fig. 2; 62 and 19\% of the hybridization spots were localized on the Ip13 and Ip21 bands, respectively. 
From these data we conclude that the human CD53 gene maps to Ip13, proximal to Ip21.

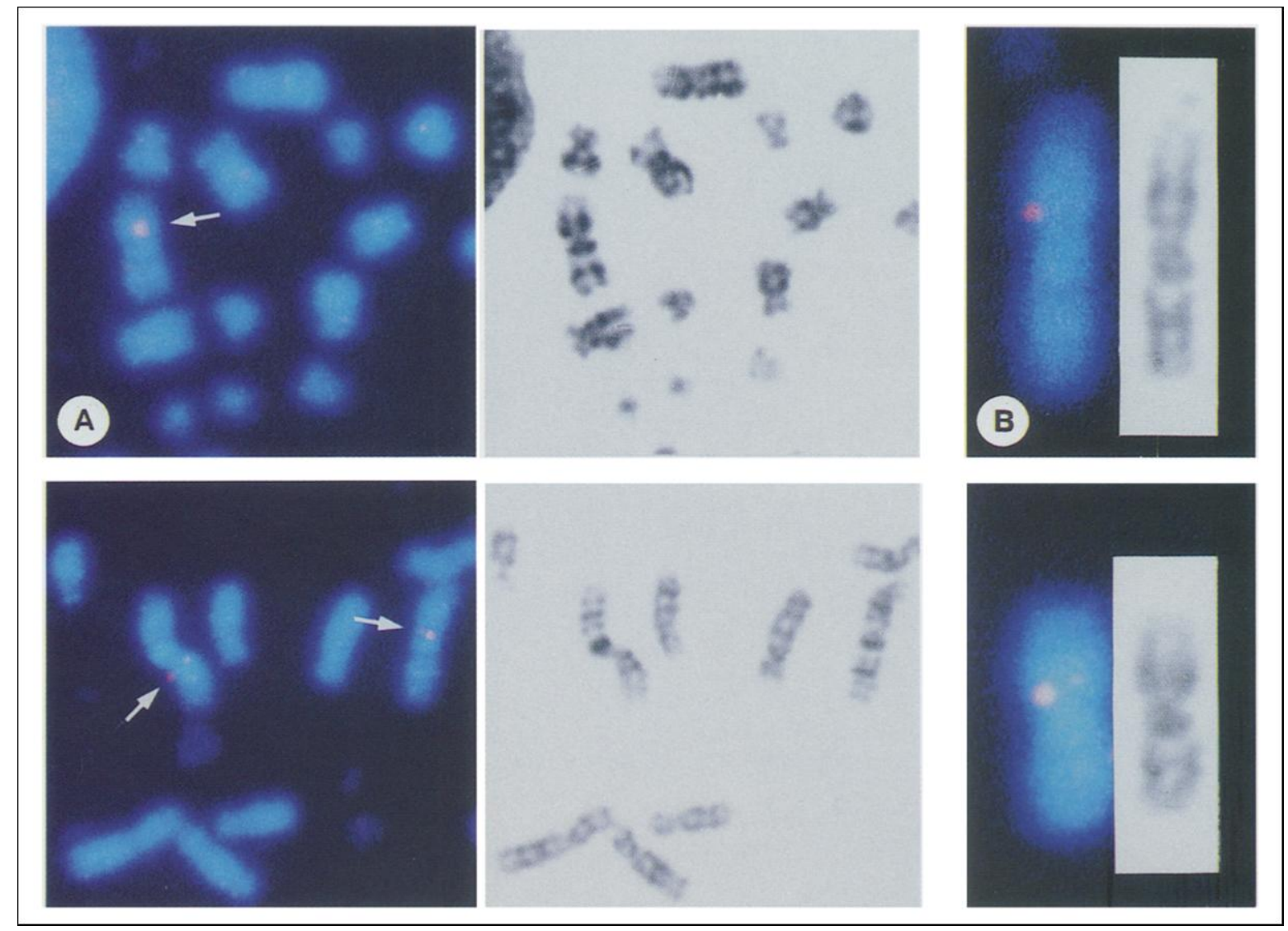

FIG. 1. Fluorescence in situ hybridization on human chromosomes with a digoxigenin-labeled 17-kb-long genomic DNA probe of the human CD53 gene. Arrows indicate the specific site of hybridization. (A) Rhodamine detection of digoxigenin-labeled probe and DAPI counterstaining

in human mitotic cells and GTG-banding of the same metaphase spread. (B) Examples of chromosome 1 from other cells.

Chromosomal region Ip13 contains some other lymphoid genes like CD2 and its ligand CD58 (12). Whether the three lymphoid genes are linked, forming a cluster, is currently unknown. Interestingly, CD53 and. CD2 antigens coprecipitate with antibodies against CD53 in rat T lymphocytes and natural killer cells, suggesting that they are associated on the cell membrane (3). Furthermore, in these cells the response to antigenic stimulation of $\mathrm{CD} 2 \div$ cells is increased severalfold if they are CD53 $\div(3)$. These observations raise the possibility that these two genes might have a coordinated expression in T-cells. This interaction and the pattern of OX44/CD53 expression at different stages of hematopoietic development and in very different mature cell types make the regulation of CD53 gene transcription an interesting problem. Chromosomal translocations specific for leukemias have been very instrumental in the discovery of genes implicated in oncogenesis. There are some translocations involving the Ip13 region in different types of tumors (7). 
Among them is $t(1 ; 22)$ ( $p 13 ; q 13)$, which is detected in $70 \%$ of the cases of $M 7$ acute megakaryocytic leukemia in young children and has a fatal prognosis (6). Because of the possible effects of CD53 on cell proliferation, it might be implicated in the oncogenic phenotype. Two RAS-related genes (10), NRAS and RAPIA, and other genes, such as NGFB and TSH, which might have effects on the growth properties of a cell, also map to Ip13. Which genes are affected by the $t(1 ; 22)$ $(p 13 ; q 13)$ translocation is not known, but a better characterization of this region should help to clarify this point.

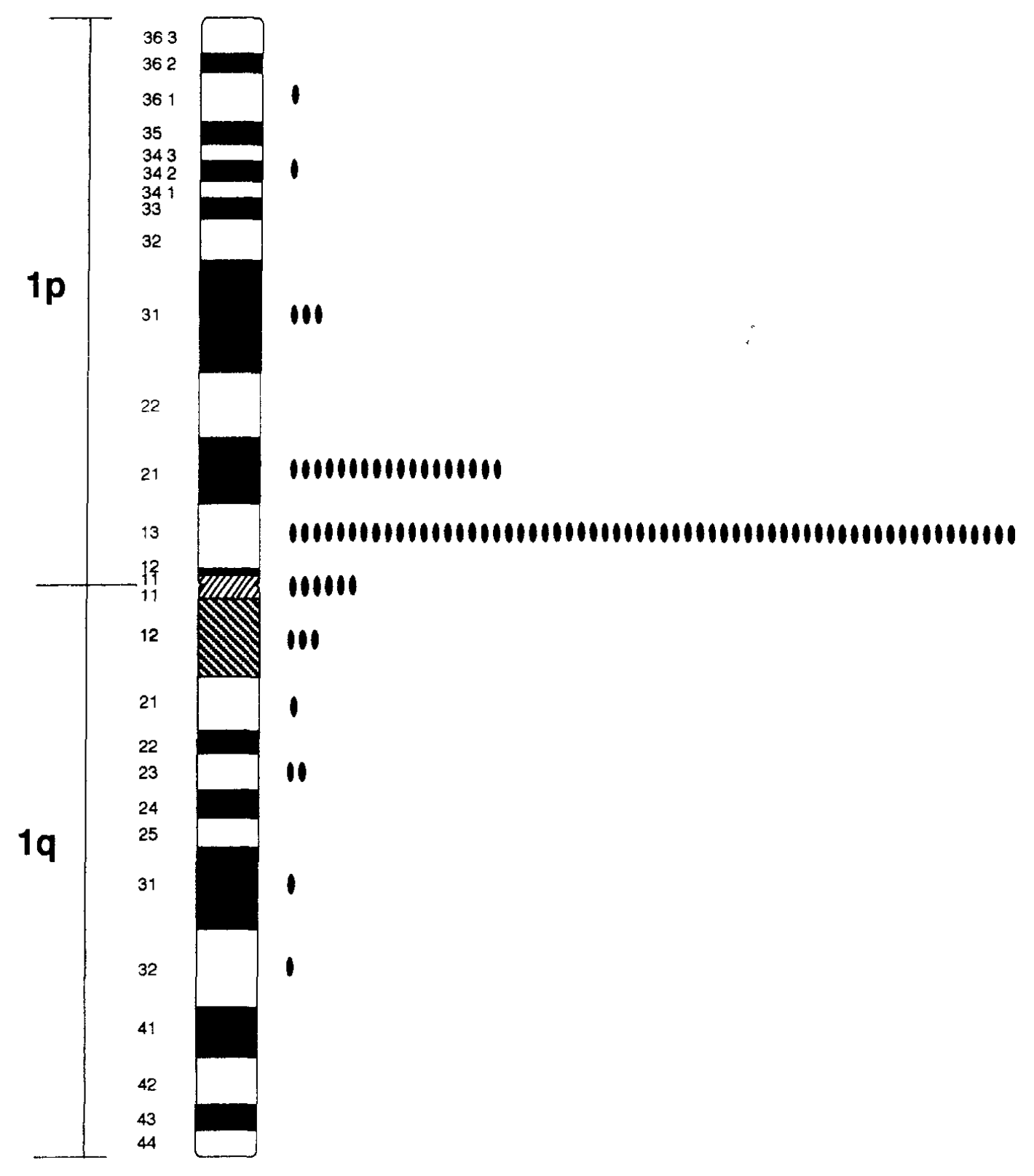

FIG. 2.

Assignment of the CD53 gene to Ip13. The idiogram of human chromosome 1 schematically indicates the intrachromosomal 
ACKNOWLEDGMENTS:

This work has been supported by grants from Fundación Ramón Areces and CICYT (SAL90-0209 and SAL91-0043) to P.A.L. and FIS (92-0889) to S.R.C. 